Tél. 80.63 .30 .02

\title{
États de l'azote organique et devenir de l'azote apporté sous les prairies de moyenne montagne
}

\author{
P Loiseau 1, C Grignani 2 \\ avec la collaboration technique de G Merle 1, M Rousseau ${ }^{1}$, G Bielicki 1 \\ 1 INRA, station d'agronomie, laboratoire des productions fourragères, 63039 Clermont-Ferrand, France; \\ 2 Istituto di scienza delle coltivazioni, 10126 Turin, Italie
}

(Reçu le 16 août 1989; accepté le 18 janvier 1991)

\begin{abstract}
Résumé - Les matières organiques du sol sont étudiées sous des prairies de montagne différenciées par la gestion agronomique. Les quantités de carbone sont plus importantes sous pelouses pauvres que sous pelouses améliorées ou prairies riches alors que les quantités d'azote sont moins variables d'une prairie à l'autre. Le fractionnement granulométrique des matières organiques montre une accumulation de débris grossiers à rapport [C]/[N] élevé sous pelouse pauvre. Le marquage des différentes fractions organiques est examiné 2 ans après un apport de 15N: l'azote apporté à la pelouse améliorée est retrouvé en plus grande partie dans les fractions fines et celui apporté à la pelouse pauvre pour moitié dans les fractions fines et grossières. Dans les 2 sites, le rapport d'allocation de l'azote marqué est élevé dans les fractions grossières qui contiennent les racines et des débris morts. Les bilans de $15 \mathrm{~N}$ sont présentés. Il n'y a pas de liaison négative entre le recouvrement de l'azote par le sol et par le peuplement épigé; le défaut de bilan augmente avec la date d'apport. Le rôle de la carence en $\mathrm{N}$ minéral sur l'accumulation des matières organiques grossières dans la pelouse pauvre est discuté. La pelouse améliorée résulte de l'investissement de quantité minimes d'azote en 15 ans sur la pelouse pauvre.
\end{abstract}

azote ${ }^{15} \mathrm{~N}$ / immobilisation / fraction organique / prairie / réorganisation

Summary - Status of organic nitrogen and fate of mineral nitrogen in mountain pastures with high soil organic matter content. Soil organic matter was investigated in mountain pastures selected for their contrasted intensification. The amounts of carbon were greater under poor $(P)$ than intensified swards $(M)$ or meadows $(R)$ : respectively 212, 182 and $164 \mathrm{t}^{\mathrm{h}} \mathrm{a}^{-1}$ (C) for $30 \mathrm{~cm}$ of soil. In contrast, the amounts of nitrogen were less variable: 18.2, 16.1 and 14.9 t ha $^{-1}(\mathrm{~N})$. Organic matter separation according to particle size demonstrated the accumulation of coarse organic fragments with high $\mathrm{C} / \mathrm{N}$ ratios (fig 4) under poor sward (fig 2). The labelling of the diverse organic fractions was examined 2 yr after application of $15 \mathrm{~N}$. The major percentage of nitrogen applied was found in the fine fraction of the intensified sward, and half in each of the fine and coarse fractions of the poor sward (fig 5). At the 2 sites, the ${ }^{15} \mathrm{~N}$ extractability index was high in the coarse fractions, which included roots and dead fragments (table I). The ${ }^{15} \mathrm{~N}$ balance is shown in table II. There was no negative relation between nitrogen uptake by the soil and the aerial parts of the sward. The lack of balance increased with the date of ${ }^{15} \mathrm{~N}$ application. The role of mineral $\mathrm{N}$ deficiency on organic matter accumulation is discussed. The intensified sward resulted from an improvement of the poor sward by minor annual mineral $N$ investments over a 15-yr period.

${ }^{15}$ nitrogen / organic fraction / permanent grassland / immobilisation / reorganisation

\section{INTRODUCTION}

Les pelouses et prairies de montagne développées sur andosols couvrent de vastes surfaces dans le Massif central français. Leur typologie a montré les relations étroites entre l'état du sol, la composition botanique et la production épigée (de Montard et Gachon, 1978). Les pelouses les plus pauvres sont caractérisées par de très fortes accumulations de matières organiques dans le sol. La liaison négative entre la production fourragère et l'accumulation du carbone dans le sol suggère un blocage du cycle de l'azote. Un article précédent (Loiseau, 1989) a chiffré la fourniture en $\mathrm{N}$ du sol à $29 \mathrm{~kg} \cdot \mathrm{ha}^{-1} \cdot \mathrm{an}^{-1}$ sous pelouse pauvre et $75 \mathrm{~kg} \cdot \mathrm{ha}^{-1}$.an-1 sous pelouse amélio- 
rée, et les coefficients réels d'utilisation des apports minéraux de $\mathrm{N}$ à $30 \%$ sous pelouse pauvre et $39 \%$ sous pelouse améliorée. Pour expliquer ces chiffres, on peut avancer l'hypothèse de travail suivante : dans des milieux à forte accumulation de carbone organique, la minéralisation des matières organiques est limitée par la disponibilité en azote minéral. On pourrait définir des besoins du sol en azote minéral, au même titre que des besoins du peuplement végétal, et par conséquent une compétition entre sol et peuplement pour l'utilisation de l'azote minéral. Les besoins du sol n'étant pas satisfaits, la minéralisation est faible et limite la disponibilité en azote minéral pour l'évolution du sol comme pour la croissance des plantes.

Une série d'investigations a été entreprise pour préciser la dynamique de l'azote dans les systèmes sol-peuplement prairial et les formes de stockage de l'azote dans les matières organiques, en utilisant une gamme de prairies contrastées. Les études relatées ici concernent :

- la caractérisation des stocks organiques azotés du sol par le fractionnement des matières organiques;

- l'incorporation de l'azote minéral non utilisé pour la croissance épigée dans les stocks organiques du sol;

- un bilan sur le devenir des apports minéraux.

\section{MATÉRIEL ET MÉTHODES}

\section{Sites prairiaux}

Les investigations ont été réalisées dans 2 types d'écosystèmes pâturés dérivant d'un même état initial : un témoin resté à l'état de pelouse peu exploitée par le bétail et une pelouse identique à l'origine, mais exploitée depuis 15 ans en pâturage tournant, sous un chargement supérieur à $1200 \mathrm{~kg} \cdot \mathrm{ha}^{-1} \cdot \mathrm{an}^{-1}$ (PV) et recevant $80 \mathrm{~kg} \cdot \mathrm{ha}^{-1} \cdot \mathrm{an}^{-1}$ ( $\mathrm{N}$ minéral). Pour certaines études, un $3^{e}$ sîte a été ajouté sous la forme d'un écosystème de prairie permanente de fauche, située dans les mêmes conditions de sol mais développée après un passé agricole ancien de culture. Les 3 écosystèmes correspondent à des intensifications croissantes (fertilisation en $\mathrm{N}$, production et utilisation). Ils sont localisés à des distances voisines mais à des altitudes de 1350,1200 et $1050 \mathrm{~m}$ respectivement pour la pelouse pauvre $(P)$, la pelouse intensifiée $(M)$ et la prairie de fauche $(R)$. Les types de sols (andosols) ont été décrits précédemment (Loiseau, 1989).

\section{Marquage des sols par ${ }^{15} \mathrm{~N}$}

Un essai avec apport d'azote minéral marqué sur les ions $\left(\mathrm{NH}_{4}{ }^{+}\right.$et $\left.\mathrm{NO}_{3}{ }^{-}\right)$avec un excès de $15 \%$ de ${ }^{15} \mathrm{~N}$ a été suivi pendant 3 ans. L'azote est apporté à 3 reprises en mai, juin et août sur $\mathrm{M}$, et à 2 reprises en juin et juillet sur $\mathrm{P}$, à raison de $50 \mathrm{~kg}(\mathrm{~N}) / \mathrm{ha}$ par apport, sous forme de $\mathrm{NH}_{4} \mathrm{NO}_{3}$. Les conditions expérimentales ont été détaillés ailleurs (Loiseau, 1989).

\section{Fractionnement des sols}

Les sols sont prélevés en début de végétation (juin), et 2 ans après les premiers apports. Trois échantillons de $5 \mathrm{~cm}$ de profondeur et $10 \mathrm{~cm}$ de côté sont prélevés par découpage au couteau tous les $5 \mathrm{~cm}$ jusqu'à 30 $\mathrm{cm}$ de profondeur, et 3 derniers entre 30 et $45 \mathrm{~cm}$ par des cylindres emporte-pièce. Après séchage à l'air, un tamisage à sec est pratiqué dans des tamis de $2 \mathrm{~mm}$ en présence d'un cylindre en fonte de $700 \mathrm{~g}$. Ce cylindre isole une fraction de débris racinaires et de graviers et permet la mesure de la densité de la terre fine. les 3 échantillons sont ensuite regroupés. Dans un premier essai de fractionnement physique, les terres tamisées à $2 \mathrm{~mm}$ ont été broyées puis séparées par densimétrie au bromoforme. Mais la méthode a été abandonnée car les fractions obtenues ne différaient pas par leur rapport [C]/[N]. La séparation chimique a été rejetée en faveur d'un autre fractionnement physique, le fractionnement granulométrique (Williams, 1983; Triessen et al, 1984), selon une procédure adaptée de Bruckert (1979). Soixante dix grammes de sol sont agités dans $400 \mathrm{ml}$ d'eau pendant $15 \mathrm{~h}$ dans un flacon de $500 \mathrm{ml}$ en présence de 10 billes de verre, puis tamisés en utilisant 5 I d'eau distillée. Les seuils de fractionnement sont de 500, 200 et $50 \mu \mathrm{m}$. Les filtrats sont séchés et pesés, et les eaux de filtration sont décantées par sédimentation dans des bidons de $5 \mathrm{I}$. Le culot est remis en allonge de sédimentation pour obtenir après évaporation le total de la fraction dont les éléments sont de taille $<2 \mu \mathrm{m}$ et la fraction d'éléments de 2 à $50 \mu \mathrm{m}$. L'ensemble des échantillons est analysé pour le carbone par la méthode Anne, et pour l'azote par la méthode Kjeldahl. Les analyses ${ }^{15} \mathrm{~N}$ ont été réalisées à la station de Laon sous la responsabilité de B Mary avec un spectromètre VG/SIRA 9.

\section{RÉSULTATS}

\section{Stocks organiques et azotés du sol}

Les sols sont caractérisés par de faibles densités apparentes et de fortes teneurs en matières or- 
ganiques. La densité du sol augmente rapidement avec la profondeur, et des pelouses à la prairie (fig 1). L'augmentation de la masse volumique sur les premiers $15 \mathrm{~cm}$ de sol est de 0,25 à $0,35(P), 0,35$ à $0,50(M)$ et 0,55 à $0,75 \mathrm{~kg} \mathrm{dm}^{-3}$ (R).

Sur $30 \mathrm{~cm}$ de profondeur, les quantités de carbone du sol décroissent avec l'intensification : 212, 182 et 164 t.ha $^{-1}$ (C) respectivement pour $P, M$ et $R$. Le carbone est réparti différemment selon les fractions granulométriques (fig 2) et les profondeurs (fig 3 ). Les fractions grossières dont la taille des éléments est supérieure à $200 \mu \mathrm{m}$ sont surtout localisées en surface et leur importance décroît avec l'intensification : 45,4, 30,1 et 8,2 t.ha $^{-1}$ (C) pour $P, M$ et $R$ (fig 3a). Les fractions organiques dont la taille des éléments se situe entre 50 et $200 \mu \mathrm{m}$ sont réparties sur tout le profil (fig $3 b$ ). Leur importance décroît avec l'intensification : 93,3, 77,2 et 47,3 tha-1 (C) pour $P$, $M$ et $R$. Les fractions dont la taille des éléments se situe entre 2 et $50 \mu \mathrm{m}$ augmentent avec l'intensification : $66,1,74,1$ et 101,1 t.ha $^{-1}$ (C) pour $P, M$ et $R$. Elles distinguent bien les sols des 3

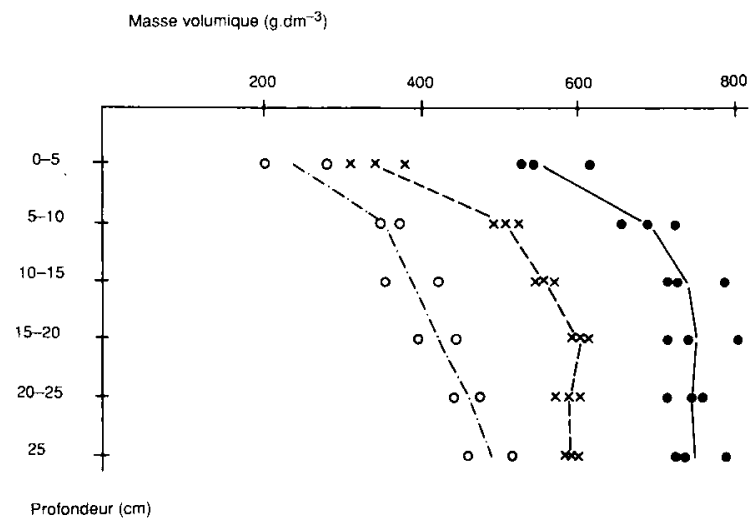

Fig 1. Profil de masse volumique du sol selon la profondeur $(\mathrm{cm}) . \longrightarrow$ prairie $(\mathrm{R}), \mathrm{x}---x$ pelouse intensifiée $(\mathrm{M})$, o-- pelouse pauvre $(P)$.

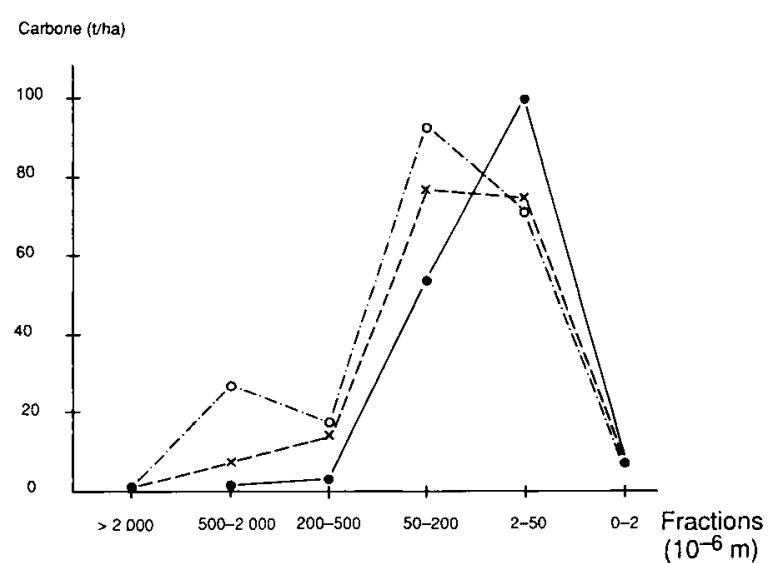

Fig 2. Quantités de carbone selon les fractions granulométriques $\left(t\right.$.ha ${ }^{-1}$ pour $30 \mathrm{~cm}$ de sol). $\longrightarrow$ prairie $(R), x--x$ pelouse intensifiée $(M), 0$... pelouse pauvre $(P)$. prairies par la quantité de limons fins dans les 15 premiers $\mathrm{cm}$ (fig $3 \mathrm{c}$ ) : les pelouses sont peu pourvues, alors que la prairie montre une répartition régulière sur tout le profil (sol isohumique).

Les quantités totales d'azote sur les $30 \mathrm{~cm}$ de sol sont respectivement de $18,2,16,1$ et 14,9 t.ha-1 $(N)$ pour $P, M$ et $R$. Comme les quantités de carbone correspondantes diminuent plus vite en fonction du site, les rapports $[\mathrm{C}] /[\mathrm{N}]$ moyens décroissent de la pelouse à la prairie, avec 11,7 , 11,3 et 11,0 pour $P, M$ et $R$. La diminution du rapport $[C] /[N]$ moyen avec l'intensification résulte de la proportion croissante de la fraction argileuse $(0-2 \mu \mathrm{m})$ à rapport $[\mathrm{C}] /[\mathrm{N}]$ bas. En effet, les rapports $[\mathrm{C}] / \mathrm{N}]$ des différents stocks organiques dépendent beaucoup plus du degré de fractionnement que de la profondeur ou du site (Cameron et Posner, 1979; Tiessen et al, 1984): les fractions à éléments grossiers de taille supérieur à $500 \mu \mathrm{m}$ ont des rapports $[\mathrm{C}] / \mathrm{N}]$ compris entre 13,8 et 20,7 ; ce rapport se situe pour les fractions de limon grossier entre 11,1 et 12,4 et pour les fractions de limon fin entre 9,2 et 11,8 . Les fractions argileuses, en très faibles quantité, montrent les rapports [C]/[N] les plus faibles entre 7,4 et 8,2 . En deuxième lieu seulement, les rapports $[\mathrm{C}] /[\mathrm{N}]$ d'une même fraction sont influencés par le site : contre toute attente, ils ne sont pas les plus élevés sur la pelouse

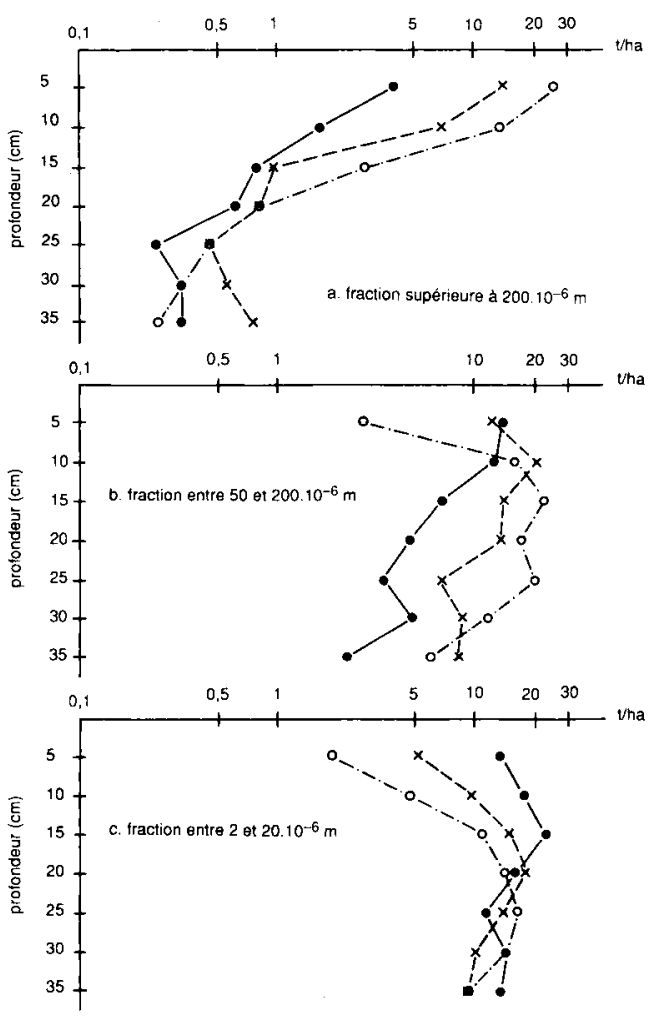

Fig 3. Répartition du carbone dans le profil de sol selon les fractions granulométriques (t.ha-1). $\longrightarrow$ prairie $(R), x--x$ pelouse intensifiée $(M), 0---$ pelouse pauvre $(P)$. 
pauvre mais sur la prairie riche; les différences dues au site sont les plus sensibles pour les rapports $[\mathrm{C}] /[\mathrm{N}]$ de fractions grossières (fig 4).

L'ensemble de ces résultats montre que l'intensification permet un fractionnement plus poussé des débris organiques. Le fractionnement physique s'accompagne d'une évolution des matières organiques qui diminue leur rapport $[\mathrm{C}] /[\mathrm{N}]$. Par conséquent, les pelouses souffrent d'un blocage de l'évolution des matières organiques qui permet l'accumulation des fractions grossières en surface et des fractions incomplètement humifiées en profondeur. Le stockage du carbone sous des formes grossières ou peu évoluées modifie en retour les conditions physiques : en particulier, la faible densité du sol semble préjudiciable à l'évolution des matières organiques (Loiseau et Martin-Rosset, 1989), peut être à travers les conditions d'humectation par remontées capillaires.

\section{Recouvrement par le sol de l'azote apporté}

On a mesuré pour les 2 pelouses, les excès isotopiques en ${ }^{15} \mathrm{~N}$ de l'azote total contenu dans les différentes fractions et profondeurs du sol, au printemps de la deuxième année suivant les apports. Les proportions totales d'engrais apporté retrouvées dans les 45 premiers $\mathrm{cm}$ de sol sont toutes situées entre 29 et $38 \%$ (fig $5 \mathrm{a}, \mathrm{b}$ ). Elles varient beaucoup selon la fraction granulométrique, la profondeur (fig 6) et le site considéré, alors que la date d'apport joue un rôle minime.

Dans le sol de la pelouse intensifiée, l'azote de l'engrais est retrouvé en plus grande partie dans la fraction humifiée de 2-50 $\mu \mathrm{m}$ (fig 5a). Dans la pelouse pauvre, il est partagé à peu près pour moitié entre la fraction humifiée et la

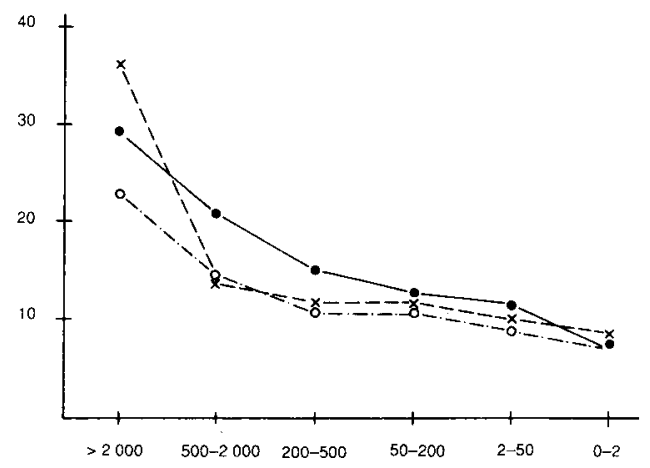

Fig 4. $\mathrm{C} / \mathrm{N}$ des matières organiques selon les fractions granulométriques $(30 \mathrm{~cm}$ de sol). - prairie $(R), x-\cdots x$ pelouse intensifiée $(M), 0---$ pelouse pauvre $(P)$.

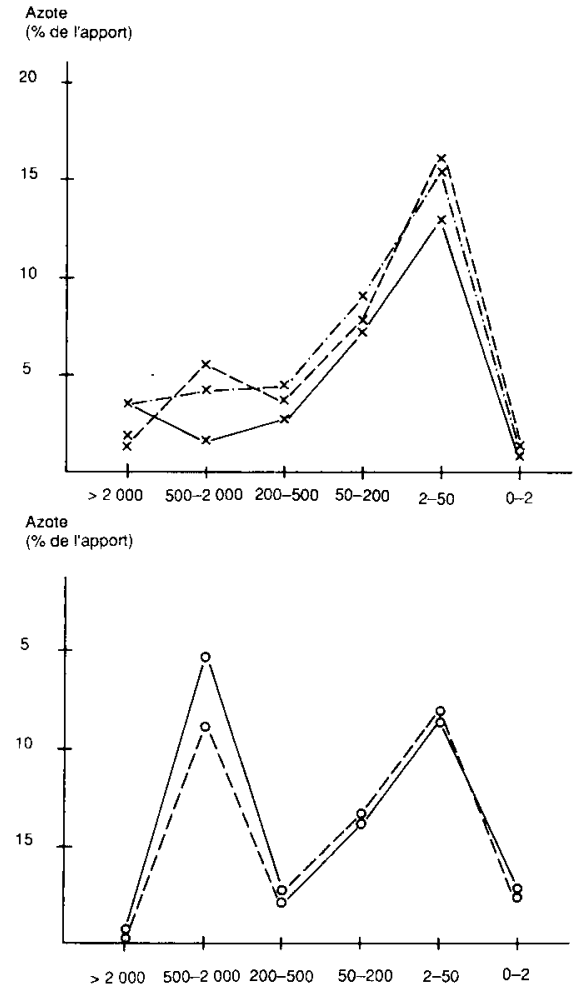

Fig 5. Part de l'apport d'engrais retrouvée dans $45 \mathrm{~cm}$ de sol, selon le site, les fractions et les dates d'apport. a) pelouse intensifiée (M); - apports de mai (total $29,1 \%$ ), - - juin (total $35,9 \%$ ) et -o- août (total $36,3 \%)$; b) pelouse pauvre; $\frac{1}{t}$ apports de mai (total $38,1 \%$ ) et juillet (total $35,1 \%$ ).

fraction grossière de 200-500 $\mu \mathrm{m}$ (fig $5 \mathrm{~b}$ ). L'azote de l'engrais se retrouve essentiellement en surface mais le gradient selon le profil est plus accusé dans la pelouse pauvre : $52 \%$ de Fractions $\left(10^{-6} \mathrm{~m}\right)$
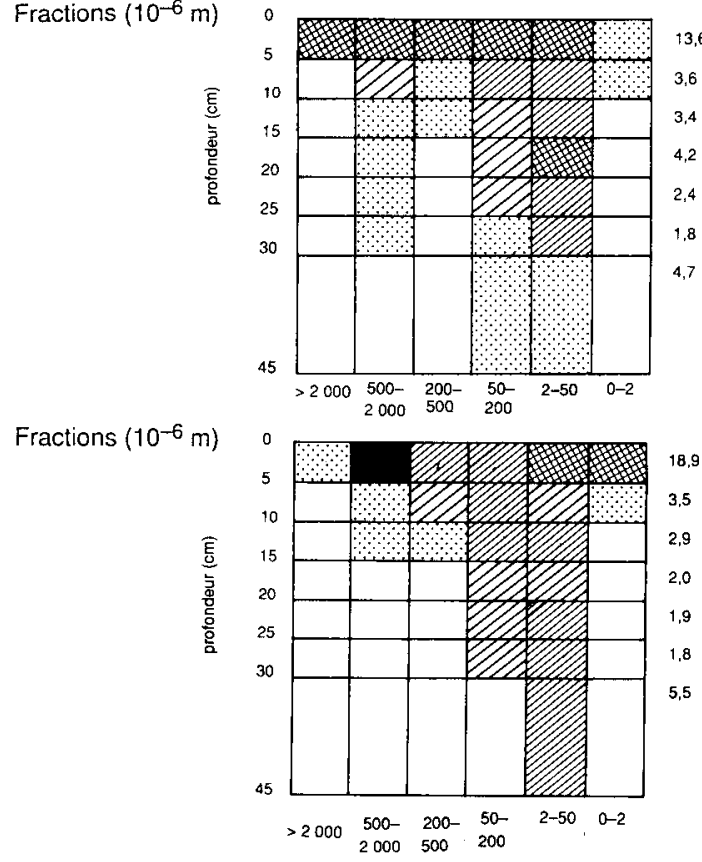

Fig 6. Part de l'apport d'engrais retrouvée dans le sol selon le site, la fraction granulométrique et la profondeur (moyenne des dates d'apports). a) pelouse intensifiée $(M)$; b) pelouse pauvre $(P)$. > 3\%; 2-3\%; [U] 1-2\%; [D 0.5-1,0\%; $0.1-0.5 \% ; \square<0.1 \%$ 
l'azote de l'engrais resté dans le sol se retrouvent dans les 45 premiers $\mathrm{cm}$ sur $\mathrm{P}$ contre $40 \%$ sur $\mathrm{M}$ (fig $6 \mathrm{a}$ et $\mathrm{b}$ ). La localisation en profondeur de l'azote issu de l'engrais se réalise surtout à travers les fractions fines de $2-200 \mu \mathrm{m}$, et beaucoup moins dans les fractions grossières de 500-2000 $\mu \mathrm{m}$. Le deuxième cas se produit néanmoins dans la pelouse intensifiée, mais pas dans la pelouse pauvre.

Les apports tardifs donnent lieu à une plus forte réorganisation totale de l'apport par le sol de la pelouse intensifiée. Cette réorganisation est en partie le fait des matières organiques grossières pour lesquelles un léger pic se dessine (fig $5 a)$.

Soient $N_{i}$ et $N_{E i}$ les quantités d'azote totales et provenant de l'engrais dans la fraction $i$. Le terme

$$
\left(N_{E i} \times \Sigma N_{i}\right) /\left(N_{i} \times \Sigma N_{E i}\right)
$$

est le rapport d'allocation (extractibility index de Janssen, 1984). Il est aussi égal au rapport de l'excès isotopique ${ }^{15} \mathrm{~N}$ de la fraction à l'excès ${ }^{15} \mathrm{~N}$ du sol tout entier. II prend la valeur 1 pour le sol entier, des valeurs $>1$ si la fraction contient une plus forte proportion d'azote de l'engrais que la moyenne du sol, et $<1$ dans la cas inverse. Les fractions humifiées de 2-50 $\mu \mathrm{m}$, qui contiennent une grosse partie de l'azote ont des valeurs voisines de 1. La fraction peu humifiée de 50-200 $\mu \mathrm{m}$ a un rapport d'allocation plus faible que la moyenne. Les fractions grossières dont les particules ont une taille $>500 \mu \mathrm{m}$ ont un rapport d'allocation très élevé (tableau I).

\section{DISCUSSION}

\section{Rétention d'azote issu de l'engrais dans les fractions organiques}

La rétention d'azote de l'engrais par le sol, $36 \%$ sur $\mathrm{P}$ et $33 \%$ sur $\mathrm{M}$, correspond aux chiffres les plus élevés de la littérature, soit $13-26 \%$ de l'apport pour des prairies permanentes riches à base de ray grass anglais recevant $100 \mathrm{~kg}(\mathrm{~N}) /$ ha (Whitehead, 1983; Whitehead et Dawson, $1984), 32 \%$ ou $31-35 \%$ sous ray grass anglais recevant $400 \mathrm{~kg}(\mathrm{~N}) /$ ha (Colbourn, 1983; Webster et Dowdell, 1985), 40\% sur fétuque élevée (Triboi, 1987). La rétention est très forte dans les 5 premiers $\mathrm{cm}: 40(\mathrm{P})$ et $51 \%(\mathrm{M})$ de la rétention totale par le sol, par rapport à $27-39 \%$ (Webster et Dowdell, 1985), ou 24\% (Bristow et al, 1987).

Une partie du $15 \mathrm{~N}$ retrouvé dans les fractions fines peut dériver de corps microbiens marqués vivants ou humifiés. Mais le marquage sur tout le profil, 21 mois après le dernier apport marqué (fig 6) suggère une stabilisation directe, physique ou chimique (Tiessen et al, 1984) d'azote dans les complexes humiques après entraînement en profondeur des nitrates en phase liquide. Ce phénomène, commun aux 2 pelouses, est plutôt passif (rapport d'allocation faible), mais important compte tenu du stock d'azote.

La fixation ou l'organisation d'azote minéral dans la fraction grossière est importante (fort rapport d'allocation) et variable en fonction de la

Tableau I. Rapport d'allocation du ${ }^{15} \mathrm{~N}$ dans les fractions granulométriques de la matière organique des sols. Chiffres pour la moyenne des périodes d'apports, et pour $45 \mathrm{~cm}$ de sol 2 années après l'apport.

\begin{tabular}{|c|c|c|c|c|c|c|}
\hline $\begin{array}{l}\text { Fraction (taille } \\
\text { des éléments en } \mu \mathrm{m} \text { ) }\end{array}$ & $>2000$ & $500-2000$ & $200-500$ & $50-200$ & $2-50$ & $<2$ \\
\hline $\begin{array}{l}\text { Pelouse pauvre } \\
\text { Apport de: }\end{array}$ & & & & & & \\
\hline $\begin{array}{l}\text { juin } \\
\text { juillet }\end{array}$ & $\begin{array}{l}5,3 \\
4,0\end{array}$ & $\begin{array}{l}4,6 \\
4,6\end{array}$ & $\begin{array}{l}1,1 \\
1,1\end{array}$ & $\begin{array}{l}0,4 \\
0,5\end{array}$ & $\begin{array}{l}0,8 \\
0,8\end{array}$ & $\begin{array}{l}1,6 \\
1,6\end{array}$ \\
\hline $\begin{array}{l}\text { Pelouse riche } \\
\text { Apport de: }\end{array}$ & & & & & & \\
\hline $\begin{array}{l}\text { juin } \\
\text { juillet } \\
\text { août }\end{array}$ & $\begin{array}{l}19,3 \\
16,7 \\
28,3\end{array}$ & $\begin{array}{l}3,9 \\
3,8 \\
5,0\end{array}$ & $\begin{array}{l}1,7 \\
1,5 \\
2,3\end{array}$ & $\begin{array}{l}0,6 \\
0,6 \\
0,7\end{array}$ & $\begin{array}{l}1,0 \\
1,0 \\
0,8\end{array}$ & $\begin{array}{l}0,5 \\
0,6 \\
0,8\end{array}$ \\
\hline
\end{tabular}


date d'apport, du type de pelouse et de la profondeur. Une interprétation sur la nature de la fraction devient nécessaire. La nature essentiellement organique de la fraction dont la taille des éléments est $>500 \mu \mathrm{m}$ est montrée par sa teneur en carbone : $26-30 \%$ sur $P ; 40-42 \%$ sur $M$. Les stocks d'azote dans la fractions organiques grossières à élément de taille $>500 \mu \mathrm{m}$ sont de $1590(\mathrm{P})$ et $450(\mathrm{M}) \mathrm{kg}(\mathrm{N}) / \mathrm{ha}$. Selon un examen à la loupe, ces stocks se composent de débris à structure organique plus ou moins altérée mais encore reconnaissable et d'agrégats de débris végétaux très altérés, jaunis et de structure subsphérique. D'après leur aspect, ces matières organiques sont plus altérées sur $P$ que sur $M$. Elles sont aussi plus riches en azote (fig 4). Les 2 sols se différencient donc par l'abondance, le rapport $[\mathrm{C}] /[\mathrm{N}]$ et probablement par l'âge de cette matière organique jeune, dont le temps de renouvellement pourrait varier entre $10(P)$ et 1 an (R) (Janssen, 1984). Cette accumulation est aussi liée au pH (Miki et Takao, 1984).

Une extraction sélective des racines par lavage de sol frais donne des chiffres moyens de l'ordre de $380(\mathrm{P})$ et $500(\mathrm{M}) \mathrm{kg}(\mathrm{N}) /$ ha contenus dans la biomasse et une partie de la nécromasse racinaire (non publié). Ainsi, les 2 pelouses se distinguent davantage par la masse d'azote associée aux débris morts que par les masses racinaires fraîches. Par conséquent, les différences constatées entre les proportions d'azote issu de l'engrais dans les fractions grossières de $P$ et $M$ doivent être plutôt attribuées à une forte allocation de l'azote issu de l'engrais dans les débris morts rapport $[\mathrm{C}] / \mathrm{N}]$ élevé situés en surface de la pelouse pauvre. La réorganisation racinaire joue un certain rôle; elle est sans doute responsable de la rétention en profondeur d'azote minéral dans la fraction grossière de la pelouse intentisifée (Triboi, 1987). Mais le coefficient d'utilisation de l'engrais par les racines est généralement faible, même sous prairie temporaire : 10\% sur fétuque élevée (Triboi, 1987); $7 \%$ sur ray grass anglais (Whitehead et Dawson, 1984; Bristow et al, 1987). D'autres formes de réorganisation sont suceptibles de prendre place dans la fraction dont les éléments sont de taille supérieure à $200 \mu \mathrm{m}$ pour expliquer une utilisation de 11 (M) à $15 \%$ de l'engrais sur $P$. Les observations ne permettent pas de préciser la nature biologique ou chimique de cette immobilisation. En effet, les fractions ne sont pas conçues pour isoler la biomasse microbienne. Celle-ci peut se retrouver aussi bien associée à des débris ou à des complexes humifiés, ou libre dans la fraction 0-2 $\mu \mathrm{m}$. Compte tenu de son turnover rapide, on ne peut d'ailleurs observer au bout de 2 ans que le résultat de son activité.

En conclusion, les mesures ne permettent qu'une interprétation globale des phénomènes : la prairie pauvre se caractérise par une évolution et une vitesse de fractionnement lentes, doublées d'une faim d'azote minéral des apports organiques grossiers. Une liaison de cause à effet semble évidente entre la carence en azote minéral, la lente évolution des matières organiques grossières et l'accumulation du carbone en général (Whitehead, 1983).

\section{Bilan sur le devenir de l'azote apporté}

La somme des recouvrements des apports mesurés dans le sol et dans la récolte fait apparaître un défaut de bilan important et croissant avec la date d'apport (tableau II). Des défauts de bilan assez importants sont courants dans la littérature : $30 \%$ pour Colbourn (1983); $25 \%$ pour Whitehead et Dawson (1984). La lixiviation des nitrates sous la zone d'exploration racinaire existe puisque 3-6\% de l'apport est retrouvé dans la couche $30-45 \mathrm{~cm}$. Elle ne devrait pas excéder 5-8\% des apports (Colbourn, 1983; Triboi, 1987). D'autres pertes latérales sont probables, soit par drainage, soit par l'activité de la mésofaune. II faudrait aussi incriminer des pertes par volatilisation de l'ammoniac et par dénitrification peu après l'apport. Par ailleurs, la partie de l'azote restée dans les collets n'a pas été mesurée. Elle pourrait être de l'ordre de grandeur du CRU (Coefficient réel d'utilisation) de l'azote récolté l'année suivante, soit 1,8\% (M) et 2,2\% (P).

La répartition de l'azote apporté entre les parties récoltées, le sol et les pertes montre le rôle moteur prédominant des parties aériennes sur le bilan. La rétention par le sol augmente en fonction des dates d'apport, mais pas aussi vite que ne diminue la rétention dans les parties aériennes. Le pouvoir de rétention du sol, quoique très élevé, montre donc certaines limites. Contrairement à Miki et Takao (1984), les CRU dans la récolte et dans le sol ne sont pas corrélés négativement. On peut en conclure que la rétention dans le sol d'azote issu de l'engrais n'est pas non plus le simple résidu d'une rétention active dans les parties récoltées.

Les relations de compétition entre les différents compartiments du système pour l'utilisation de l'azote minéral doivent être affinées en distin- 
Tableau II. Bilan de recouvrement de l'azote apporté. Coefficient réel d'utilisation des apports azotés en \%, 2 ans après l'apport.

\begin{tabular}{lllll}
\hline & Récolte & $\begin{array}{l}\text { Sol fraction } \\
\text { grossière }\end{array}$ & $\begin{array}{l}\text { Sol fraction } \\
\text { fine }\end{array}$ & Défaut \\
\hline $\begin{array}{l}\text { Pelouse pauvre } \\
\text { Apport de juin }\end{array}$ & 38,1 & 15,4 & & \\
Apport de juillet & 15,6 & 11,5 & 22,7 & 23,8 \\
Pelouse intensifiée & & & 23,6 & 49,3 \\
Apport de mai & 62,1 & 5,2 & 23,9 & 14,0 \\
Apport de juin & 34,7 & 7,2 & 28,7 & 36,6 \\
Apport d'août & 14,5 & 6,1 & 30,2 & 55,3 \\
\hline
\end{tabular}

$1>200 \mu \mathrm{m} ;{ }^{2}<200 \mu \mathrm{m}$.

guant les fractions du sol (tableau II). Les résultats obtenus montrent une tendance à la diminution de la rétention dans les fractions fines lorsque celle des parties aériennes augmente. L'assimilation représenterait ici l'élément moteur, tandis que la rétention par les parties fines, plutôt passive ne serait qu'un simple complément. Au contraire, une véritable compétition semble exister entre les parties récoltées et les fractions grossières du sol. L'utilisation active de l'azote par ces fractions peut être attribuée aussi bien aux racines vivantes qu'aux débris organiques morts. Pour les racines, la compétition renvoie à un problème d'allocation des ressources en azote entre organes. Pour les débris, on a souligné, au moins dans la pelouse pauvre, l'existence d'une rétention nette dans les débris en voie d'humification : il y aurait donc aussi dans ce cas une compétition entre la plante entière et la fraction organique libre du sol pour l'utilisation de l'azote minéral. Mais en l'absence d'une séparation de ces 2 compartiments, il est impossible de préciser les covariations de ces flux, la nature des compétitions entre les compartiments et les priorités dans l'affectation de l'azote minéral dans le système sol-peuplement.

\section{CONCLUSION}

Sous pelouse pauvre, la minéralisation est faible. Des quantités d'azote minéral importantes sont nécessaires pour diminuer le rapport $[\mathrm{C}] /[\mathrm{N}]$ de quantités accrues de matières organiques à humifier. Cette demande n'étant pas satisfaite, l'humification n'est pas achevée et les débris s'accu- mulent sous des formes organiques spécifiques peu évoluées. Sur cet écosystème, un apport d'azote minéral ou minéralisable vient satisfaire une partie des besoins du sol. II entraîne selon toute vraisemblance une surminéralisation de carbone et peut-être d'azote, et un déblocage de l'évolution des débris restants (fractionnement, humification). Les 2 phénomènes s'ajoutent pour résorber le stock de matières organiques grossières. C'est ainsi que la pelouse intensifiée perd sur $30 \mathrm{~cm}$ de sol 18 tha de carbone par rapport à la pelouse pauvre. Cette minéralisation de carbone a été obtenue en 15 ans par une augmentation du chargement en bétail et par des apports de $80 \mathrm{~kg}(\mathrm{~N}) \cdot \mathrm{ha}^{-1} \cdot \mathrm{an}^{-1}$. Par conséquent, l'intensification, comprenant un apport d'azote minéral équivalent à $8 \%$ de la valeur initiale du stock, instaure un nouveau régime d'évolution des matières organiques dans le sol, avec augmentation de la minéralisation des apports organiques annuels, de la fourniture d'azote par le sol, et du taux d'utilisation aérienne des disponibilités en $\mathrm{N}$ minéral (Loiseau, 1989). Bien qu'absente de ce raisonnement global, la biomasse microbienne et la faune du sol jouent certainement un rôle essentiel qui resterait à préciser dans les flux entre pool minéral, matières organiques libres et humifiées.

\section{REMERCIEMENTS}

Nous remercions L Gachon qui a favorisé l'emploi du $15 \mathrm{~N}$ dans le laboratoire, B Mary, de la station d'agronomie de Laon pour son aide à l'interprétation des résultats et l'Intergroupe Azote du ministère de l'Environnement (SRETIE) pour son aide. 


\section{RÉFÉRENCES}

Bristow AW, Ryden JC, Whitehead DC (1987) The fate of several times interval of $15 \mathrm{~N}$ labelled ammonium nitrate applied to an established grass sward. J Soil Sci 38, 245-254

Bruckert S (1979) Séparation des complexes organominéraux et des matières organiques libres par tamisage dans l'eau. Ann Sci Univ Besançon Biol Vég 3, 20, 3-7

Cameron RS, Posner AM (1979) Mineralisable organic nitrogen in soil fractionated according to particle size. J Soil Sci 30, 565-577

Colbourn P (1983) Losses of fertilizer from grass sward. EEC Workshop "Nitrogen fluxes in intensive grassland systems", Wageningen, 12-14 Oct 1983, 147-149

De Montard FX, Gachon L (1978) Contribution à l'étude de l'écologie et de la productivité des pâturages d'altitude des Monts Dore. 1. Application de l'analyse factorielle des correspondances à l'analyse de la végétation. Ann Agron (Paris) 29, 277310

Janssen BH (1984) A simple method for calculating decomposition and accumulation of "young" soil organic matter. Plant Soil 76, 297-304

Loiseau P (1989) Fourniture par le sol et utilisation de l'azote minéral dans les pâturages et les prairies de montagnes volcaniques. Agronomie 9, 965-972
Loiseau P, Martin-Rosset W (1989) Évolution d'une lande de montagne pâturée par des bovins ou des chevaux. II. Production fourragère. Agronomie 9, 161-169

Miki N, Takao K (1984) Uptake of fertilizer nitrogen by cocksfoot sward with different soil acidity. Bull Hokkaido Prefect Agric Exp Stn 51, 43-53 (in Herb Abstr)

Tiessen H, Stewart JWB, Hunt HW (1984) Concepts of soil organic matter transformation in relation to organo-mineral particle size fractions. Plant Soil 76, 287-295

Triboi E (1987) Recovery of mineral $N$ fertilizer in herbage and soil organic matter in grasslands of the Massif central, France. Fert Res 13, 99-116

Webster CP, Dowdell RJ (1985) A lysimeter study of the fate of nitrogen applied to perennial ryegrass swards. Soil analysis and the final balance sheet. $J$ Soil Sci, 605-611

Whitehead DC (1983) Nitrogen dynamics in the soil under intensively managed grassland. EEC Workshop "Nitrogen fluxes in intensive grassland systems", Wageninen, Oct 1983, 68-78

Whitehead DC, Dawson KP (1984) Nitrogen, including $15 \mathrm{~N}$ labelled fertilizer nitrogen, in components of a grass sward. J Appl Ecol 21, 983-989

Williams BL (1983) The nitrogen content of particle size fractions separated from peat and its rate of mineralization during incubation. $J$ Soil Sci 34, 113124 